\title{
A SEIRD Model for Analysing the Dynamics of Coronavirus (COVID-19) Pandemic in Nigeria
}

\author{
Ashiribo S. Wusu ${ }^{1, *}$, Olusola A. Olabanjo ${ }^{2}$, Benjamin S. Aribisala ${ }^{2}$ \\ ${ }^{1}$ Department of Mathematics, Lagos State University, Lagos, Nigeria \\ ${ }^{2}$ Computer Science Department, Lagos State University, Lagos, Nigeria
}

Cite This Paper in the following Citation Styles

(a): [1] Ashiribo S. Wusu, Olusola A. Olabanjo, Benjamin S. Aribisala, "A SEIRD Model for Analysing the Dynamics of Coronavirus (COVID-19) Pandemic in Nigeria," Universal Journal of Applied Mathematics, Vol.9, No.2, pp. 10-15, 2021. DOI: 10.13189/ujam.2021.090102.

(b): Ashiribo S. Wusu, Olusola A. Olabanjo, Benjamin S. Aribisala, (2021). A SEIRD Model for Analysing the Dynamics of Coronavirus (COVID-19) Pandemic in Nigeria. Universal Journal of Applied Mathematics, 9(2), 10-15. DOI: 10.13189/ujam.2021.090102.

Copyright $@ 2021$ by authors, all rights reserved. Authors agree that this article remains permanently open access under the terms of the Creative Commons Attribution License 4.0 International License

\begin{abstract}
The first case of the novel coronavirus (COVID-19) in sub-Saharan Africa was confirmed by Nigeria and the figure has since then been on the rise. Current global efforts are geared towards getting effective vaccine for the cure of the disease. The hope of accessing the relieve offered by the arrival of such vaccine will obviously take significant amount of time. In the face of the resurgence of the disease, the need to slow the spread and flatten the curves is currently a priority of both governmental and non-governmental organisations in Nigeria. If the dynamics of the disease can be determined, then it becomes easier to strategize and make suitable preventive policies that will slow the spread and ultimately flatten the $\mathrm{c}$ urves. Here, the goal is to develop a compartmental-based model for analysing the dynamics of the pandemic in Nigeria. Considering the control policies currently in place - social distancing, mask usage, personal hygiene and quarantine, and using data provided by Nigeria Centre for Disease Control (NCDC), World Health Organization (WHO) and Wolfram Data Repository on COVID-19, the proposed model is fitted to the available data using the Quasi-Newton algorithm. The infection rate, average latent time, average infective time and average mortality rate are estimated. Also, the overall effectiveness of the current control policies is measured. Predictions on the turning points and possible vanishing time of the virus in Nigeria are made. Recommendations on how to manage the resurgence of the disease in Nigeria are also suggested.
\end{abstract}

Keywords Coronavirus, COVID-19, Pandemic, Compartmental Model, Nigeria

\section{Introduction}

Standard epidemiological models provide comprehensive pathway into the understanding of the dynamics of an epidemic outbreak [1]. The need to recommend a standard and scientific method for a global solution to a pandemic is a global concern especially in recent times due to the recent challenges of the novel CoronaVirus (COVID-19) pandemic. Compartmental models are a good direction to face in terms of epidemiological modeling especially for predicting, determining, validating and analyzing the rate of susceptibility, exposure, infection, recovery and mortality due to an infection - usually pandemic [1], [2, 3]. Although several models have been proposed and fitted by researchers, however, the assessment of the impacts of responses and determination of a number of qualitative parameters of each unique occurrence has made this study even more complex. In epidemic modeling, it is believed that the incidence rate is given by an unspecified nonlinear function constrained by a few biologically motivated conditions. The meteoric rise in the rate of spread of CoronaVirus in Nigeria has raised some social and health measures such as social distancing, lockdown, border closure, total stoppage of air-flights and banning of social gatherings among others [4]. These measures aim at reducing the contact paramter(s) [5, 6]. Epidemic models seek to predict several things such as how a pandemic spreads, the estimated number of infections, the estimated duration as well as the reproductive rate of the infection using ordinary differential equations which are deterministic in nature [7, 8, 9]. More sensitive parameters can, 
however, be included such as how different public health interventions may affect the spread including vaccine administration [10]. As the number of cases increases, there is need to understand the dynamics of the pandemic and hence, provide the best strategy to slow the spread and eventually flatten the curves. Efforts have been made to model and visualize the geographical spread of the disease $[17,19]$. In the efforts to estimate some parameters that can be used to project the severity of the outbreak, its duration, and the mortality rate, several epidemiological models [20, 21, 22, 23, 14] have been proposed. This work made use of the pandemic data in the period of January 22, 2020 to December 01, 2020 provided by the Nigeria Centre for Disease Control (NCDC), World Health Organization (WHO) and Wolfram Data Repository on COVID-19. A Susceptible-Exposed-InfectiousRecovered-Deceased (SEIRD) model for analyzing the dynamics of coronavirus (COVID-19) pandemic in Nigeria is presented. The overall effectiveness of the current control policies - social distancing, mask usage, personal hygiene and quarantine are measured. The model parameters - infection rate, average latent time, average infective time and average mortality rate are also estimated. Predictions on the turning points and possible vanishing time of the virus in Nigeria are made. Recommendations on how to manage the resurgence of a second wave of the disease in Nigeria are also suggested.

\section{Model Formulation}

To analyse the dynamics of the COVID-19 pandemics in Nigeria, we propose a compartmental model consisting of five states - Susceptible (S), Exposed (E), Infective (I), Recovered (R) and Deceased (D). The model divides the entire population into five categories. Throughout this work, we shall referred to this model as Susceptible-Exposed-Infectious-Recovered-Deceased (SEIRD) model. The Susceptible category is the set of people who could potentially get the disease, the Exposed persons are those who have contacted the disease and are in the latency period, the Infective category contains people who currently have the disease and can infect others, those who are able to recover from the disease are classified as the Recovered category and the Deceased category is made up of people who have died of the disease. This work assumes that the population size is constant and the rate of infection to be proportional to the contact. Each compartment represents a group of individuals in the same health state. The direction and rate of movement from one state to another is indicated in the connections between the compartments as seen in Figure 1. Mathematically, these connections are described by a system of ordinary differential equations

$$
\left.\begin{array}{r}
\frac{d S}{d t}=-\beta(1-\mu) S(t) \lambda(t) \\
\frac{d E}{d t}=-\gamma E(t)+\beta(1-\mu) S(t) \lambda(t) \\
\frac{d I}{d t}=\gamma E(t)-\delta I(t)-f I(t) \\
\frac{d R}{d t}=\delta I(t) \\
\frac{d D}{d t}=f I(t)
\end{array}\right\}
$$

where $\mu \in[0,1]$ is the overall measure of the effectiveness of the current control policies, $\lambda(t)=\frac{I(t)}{N-D(t)}$ is the force of infection, constant $N=S+E+I+R+D$ is the total population, $\beta, \gamma^{-1}, \delta^{-1}$ and $f$ are the infection rate, average incubation time (days), average infective time (days) and case mortality rate respectively. The term $S(t) \lambda(t)$ represents the contacts between the Susceptible and the Infected category.

\section{Parameter Estimation}

To optimally estimate the parameters of our model, (1) is solved numerically in terms of the model parameters. The dynamics of the pandemic can be visualized by varying the parameters as seen in 1 . In this work, the parameters were manually tuned to get the initial guess to be used in the curve fitting. Following parameter initialization, the resulting curves from the solution of (1) are then fitted to the available data using the Quasi-Newton algorithm. Optimal parameter values that fit the data as obtained are presented in Table 1 .

\section{Interpretation of Model Solution}

The model (1) describes the dynamics of the disease within the five (5) compartments. Optimal values of the parameters that fit the model show the rates of movements from one compartment to another. From the model solution, it can be seen that the turning point of the infection was determined to have occurred around the end of July, the mortality curve started flattening around mid October, the recovery curve is still on the rise. From Figure 1, it can be seen that effectiveness of the control measures are 


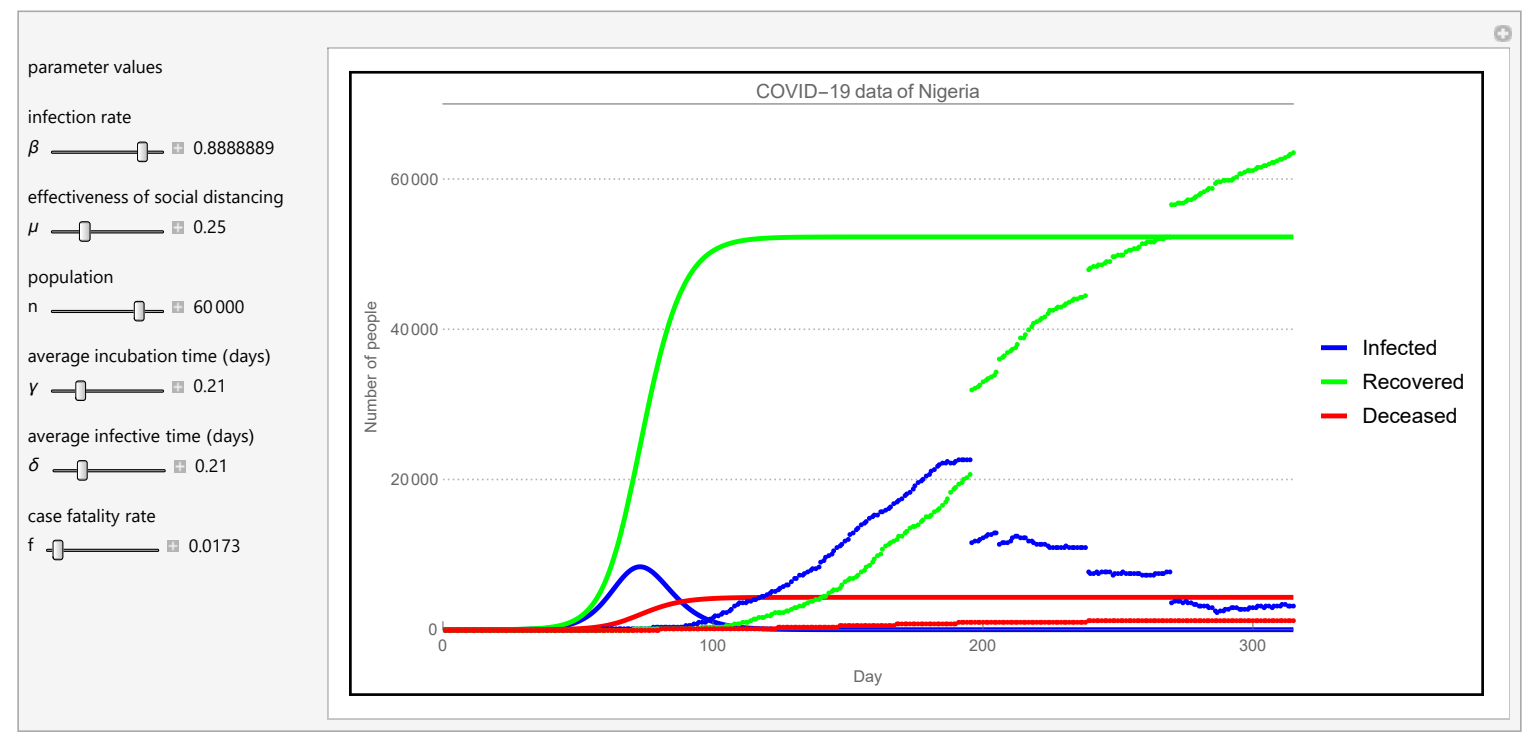

Figure 1: Model solution and parameter initialisation

\begin{tabular}{l|llll} 
& Estimate & Standard Error & t-Statistic & p-Value \\
\hline $\mathrm{N}$ & 73529.27 & 4717.115 & 15.58776 & $1.292132 \times 10^{-47}$ \\
\hline$\beta$ & 0.6652986 & 1602.972 & 0.0004150408 & 0.999669 \\
\hline$\mu$ & 0.4424032 & 1343.474 & 0.0003292979 & 0.9997373 \\
\hline$\delta$ & 0.03705205 & 0.002778987 & 13.33293 & $1.610799^{-36}$ \\
\hline$\gamma$ & 0.02913157 & 0.0003388715 & 85.96642 & 0 \\
\hline$f$ & 0.0008067493 & 0.0001394952 & 5.783349 & $1.078028 \times 10^{-8}$ \\
\hline
\end{tabular}

Table 1: Table of optimal parameter values for fitted curves

central in the quick peaking and fast-flattening of the curves. The infection rate was estimated as $\beta=0.665299$ and the overall effectiveness of the control policies was measured as $44.24 \%$. The average incubation time was estimated as $\gamma^{-1} \approx 34$ days while the average infective time was estimated as $\delta^{-1} \approx 27$ days. The mortality rate was measured as $f=0.0807 \%$. The basic reproduction number $R_{0}$ which represents the number of secondary infections in the population caused by one initial primary infection is calculated as $R_{0}=\frac{\beta}{\delta-f} \approx 18>1$. The estimated basic reproduction number $R_{0}>1$ clearly shows that there is already an epidemic.

\section{Sensitivity Analysis}

To evaluate the influence of the parameters on the long-term behaviour of the model, a sensitivity analysis is performed on the data by systematically varying the values of the parameters. The asymptotic parameter correction matrix is presented in Table 2 and the fitted curve based on the optimal parameter estimates can be seen in Figure 2.

\section{Equilibrium Solutions and Stability of Model}

In this section, we discuss the equilibrium solution and stability of the model (1). The equilibrium points are the values $S_{e}$, $E_{e}, I_{e}, R_{e}$ and $D_{e}$ of $S, E, I, R$ and $D$ respectively for which if, at a specific time $t_{k}, S=S_{e}, E=E_{e}, I=I_{e}, R=R_{e}$ and $D=D_{e}$ then $S, E, I, R$ and $D$ will remain unchanged for all $t>t_{k}$. This implies that all the model variables will remain constant and, therefore, $\frac{d S}{d t}=0, \frac{d E}{d t}=0, \frac{d I}{d t}=0, \frac{d R}{d t}=0, \frac{d D}{d t}=0$ simultaneously at $t_{k}$. One equilibrium point of (1) is 


\begin{tabular}{l|llllll} 
& $\mathrm{N}$ & $\beta$ & $\mu$ & $\delta$ & $\gamma$ & $\mathrm{f}$ \\
\hline $\mathrm{N}$ & 1. & 0.003106 & 0.003095 & 0.981845 & -0.22034 & -0.0195223 \\
\hline$\beta$ & 0.003106 & 1.000000 & 1.000000 & -0.000908 & -0.022567 & -0.001691 \\
\hline$\mu$ & 0.003095 & 1.000000 & 1.000000 & -0.000918 & -0.022561 & -0.001691 \\
\hline$\delta$ & 0.981845 & -0.000908 & -0.000918 & 1. & -0.060543 & -0.058581 \\
\hline$\gamma$ & -0.220340 & -0.022567 & -0.022561 & -0.060543 & 1.000000 & 0.056389 \\
\hline$f$ & -0.019522 & -0.001691 & -0.001691 & -0.058581 & 0.056389 & 1.000000 \\
\hline
\end{tabular}

Table 2: Asymptotic parameter correlation matrix

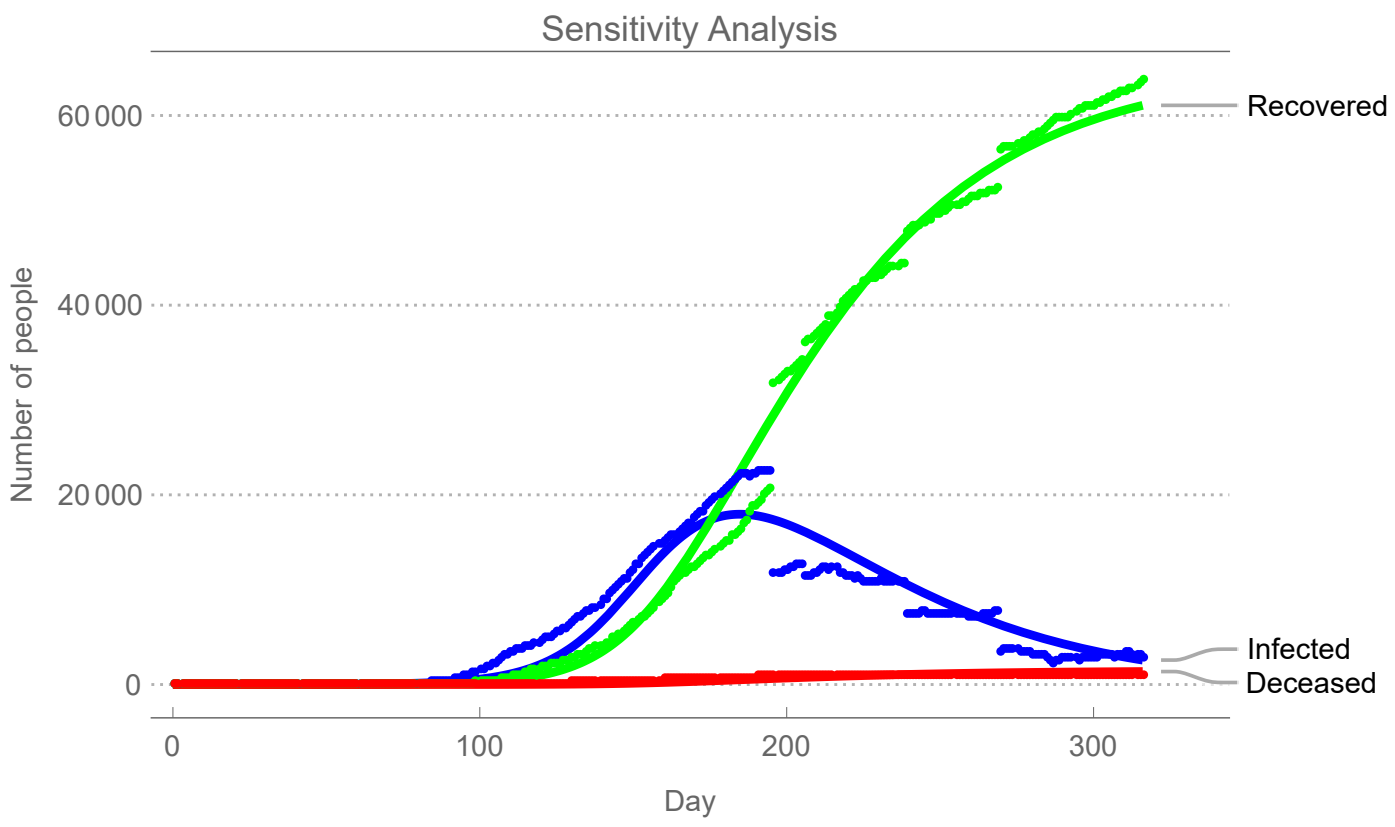

Figure 2: Sensitivity Analysis

$\left(S_{e}, E_{e}, I_{e}, R_{e}, D_{e}\right)=(0,0,0,0,0)$. To analyse the stability of the system, we linearize (1) by computing the Jacobian as

$$
J(S, E, I, R, D)=\left(\begin{array}{ccccc}
-\frac{\beta i(\mu-1)}{D-N} & 0 & -\frac{\beta(\mu-1) s}{D-N} & 0 & \frac{\beta i(\mu-1) s}{(D-N)^{2}} \\
\frac{\beta i(\mu-1)}{D-N} & -\gamma & \frac{\beta(\mu-1) s}{D-N} & 0 & -\frac{\beta i(\mu-1) s}{(D-N)^{2}} \\
0 & \gamma & -f-\delta & 0 & 0 \\
0 & 0 & \delta & 0 & 0 \\
0 & 0 & f & 0 & 0
\end{array}\right)
$$

The Jacobian at $\left(S_{e}, E_{e}, I_{e}, R_{e}, D_{e}\right)=(0,0,0,0,0)$ is given as

$$
J(0,0,0,0,0)=\left(\begin{array}{ccccc}
0 & 0 & 0 & 0 & 0 \\
0 & -\gamma & 0 & 0 & 0 \\
0 & \gamma & -f-\delta & 0 & 0 \\
0 & 0 & \delta & 0 & 0 \\
0 & 0 & f & 0 & 0
\end{array}\right)
$$

and the characteristic polynomial, $p\left(\zeta\right.$ obtained as $p(\zeta)=-\zeta^{5}+\zeta^{4}(-\gamma-\delta-f)+\zeta^{3}(\gamma(-f)-\gamma \delta)$. The roots of $p(\zeta)=0$ are obtained as $\zeta_{1}=\zeta_{2}=\zeta_{3}=0, \zeta_{4}=-\gamma, \zeta_{5}=-f-\delta$. Since $p(\zeta)=0$ has two negative roots, we conclude that the system (1) is stable. 


\section{Conclusion and Recommendation}

The SEIRD model (1) presented in this work has been used to estimate key parameters - the overall effectiveness of the control policies, infection rate, average incubation time, average infective time and the mortality rate - that are critical in understanding the dynamics of the pandemic. The deceased compartment is well fitted by the model and it can also be seen that the recovered compartment recovers almost as fast as the data. The model reveals that the overall effectiveness of the control policies in Nigeria is just $44.24 \%$. This is an indication that control policies are not properly adhered to. Based on the above, it is recommended that a strict enforcement of the control policies will significantly help manage the resurgence of a second wave of the pandemic in Nigeria.

\section{REFERENCES}

[1] Grigorieva, E., Khailov, E., and Korobeinikov, A. Optimal control for an SEIR epidemic model with nonlinear incidence rate, Studies in Applied Mathematics, Vol. 141, No. 3, pp: 353--398, (2018).

[2] Engbert, R., Rabe, M. M., Kliegl, R., and Reich, S. Sequential data assimilation of the stochastic SEIR epidemic model for regional COVID-19 dynamics, MedRxiv, (2020).

[3] Liu, Q., Jiang, D., Shi, N., Hayat, T., and Ahmad, B., Stationary distribution and extinction of a stochastic SEIR epidemic model with standard incidence, Physica A: Statistical Mechanics and its Applications, Vol. 476, pp: 58--69, (2017).

[4] Bryan T. G., Kleczkowski A., Gilligan, C. A. and Bolker B. M., Spatial heterogeneity, non-linear dynamics and chaos in infectious diseases, Statistical Methods in Medical Research, Vol. 4, No. 2, pp: 160--183, (1995).

[5] Xue, Y., Zhang, G., Pang, H., Zhou, Y., and Wang, R., Forecasting and Analysis of Time Variation of Parameters of COVID19 Infection in China Using An Improved SEIR Model, MedRxiv, (2020).

[6] Hornstein, A. Social Distancing, Quarantine, Contact Tracing, and Testing: Implications of an Augmented SEIR-Mode, Technical Report, Federal Reserve Bank of Richmond, (2020).

[7] Liu, Q., Jiang, D., Shi, N., Hayat, T., and Alsaedi, A., Asymptotic behavior of a stochastic delayed SEIR epidemic model with nonlinear incidence, Physica A: Statistical Mechanics and its Applications, Vol. 462, pp: 870--882, (2016).

[8] Sharma, N. and Gupta, A. K., Impact of time delay on the dynamics of SEIR epidemic model using cellular automata, Physica A: Statistical Mechanics and its Applications, Vol. 471, pp: 114--125, (2017).

[9] Khan, M. A., Khan, Y., and Islam, S., Complex dynamics of an SEIR epidemic model with saturated incidence rate and treatment, Physica A: Statistical Mechanics and its Applications, Vol. 493, pp: 210--227, (2018).

[10] Wang, X., Peng, H., Shi, B., Jiang, D., Zhang, S., and Chen, B., Optimal vaccination strategy of a constrained time-varying SEIR epidemic model, Communications in Nonlinear Science and Numerical Simulation, Vol. 67, pp: 37--48, (2019).

[11] Takaki, A., Wiese, M., Maertens, G., Depla, E., Seifert, U., Liebetrau, A., and Rehermann, B., Cellular immune responses persist and humoral responses decrease two decades after recovery from a single-source outbreak of hepatitis $C$, Nature medicine, Vol. 6, No. 5, pp: 578--582, (2000).

[12] Liew, F. Y., and Dhaliwal, J. S., Distinctive cellular immunity in genetically susceptible BALB/c mice recovered from Leishmania major infection or after subcutaneous immunization with killed parasites, The Journal of Immunology, Vol. 138, No. 12, pp: 4450--4456, (1987).

[13] Dantzer, R., Cohen, S., Russo, S. J., and Dinan, T. G., Resilience and immunity, Brain, behavior, and immunity, Vol. 74, pp: 28--42, (2018).

[14] Wusu A. S. and Olabanjo O. A., SEIRD Model for Analyzing Coronavirus (COVID-19) Pandemic, http://demonstrations.wolfram.com/SEIRDModelForAnalyzingCoronavirusCOVID19Pandemic/, Wolfram Demonstrations Project, Published: April 28, 2020

[15] Dantzer, R., Cohen, S., Russo, S. J., and Dinan, T. G., Resilience and immunity, Brain, behavior, and immunity, Vol. 74, pp: 28--42, (2018).

[16] Gralinski, L. E., Menachery, V. D., Return of the Coronavirus: 2019-nCoV, Viruses, Vol.(12) No.(1), pp:135, (2020). 
[17] Dong E., Du H., Gardner L., An interactive web-based dashboard to track COVID-19 in real time, Lancet Infect Dis., Vol.(20) Issue.(4), pp:533-534, (2020). DOI:https://doi.org/10.1016/S1473-3099(20)30120-1

[18] Kermack, W.O., McKendrick, A.G., A contribution to the mathematical theory of epidemics, Proc. R. Soc. Lond. A 115, pp:700-721, (1927). gallica.bnf.fr

[19] Espigule-Pons J., Mapping Novel Coronavirus COVID-19 Outbreak, https://community.wolfram.com/groups/$/ \mathrm{m} / \mathrm{t} / 1868945,(2020)$.

[20] Götz T., First attempts to model the dynamics of the coronavirus outbreak, https://arxiv.org/pdf/2002.03821.pdf, (2020).

[21] Peng L., Yang W., Zhang D., Zhuge C.and Hong L. Epidemic analysis of COVID-19 in China by dynamical modeling, https://www.medrxiv.org/content/10.1101/2020.02.16.20023465v1, (2020).

[22] Zhou Y., Chen Z., Wu X., Tian Z., Cheng L. and Ye L. The Outbreak Evaluation of COVID-19 in Wuhan District of China, https://arxiv.org/pdf/2002.09640.pdf, (2020).

[23] Jia J., Ding J., Liu S., Liao G., Li J., Duan B., Wang G. and Zhang R. Modeling the Control of COVID-19: Impact of Policy Interventions and Meteorological Factors, https://arxiv.org/pdf/2003.02985.pdf, (2020). 